\title{
1 The international and intercontinental spread and expansion of antimicrobial-resistant
}

\section{$2 \quad$ Salmonella Typhi}

4 Kesia Esther da Silva ${ }^{*}$, Arif Mohammad Tanmoy ${ }^{2,3},{ }^{*}$ Agila Kumari Pragasam ${ }^{4}$, Junaid Iqbal $^{5}$,

5 Mohammad Saiful Islam Sajib²,6, Ankur Mutreja ${ }^{7}$, Balaji Veeraraghavan ${ }^{4}$, Dipesh Tamrakar ${ }^{8}$,

6 Farah Naz Qamar ${ }^{5}$, Gordon Dougan ${ }^{9,10}$, Isaac Bogoch ${ }^{11}$, Jessica C Seidman ${ }^{12}$ Jivan Shakya ${ }^{13,14}$,

7 Krista Vaidya ${ }^{13}$, Megan E. Carey ${ }^{10}$, Rajeev Shrestha ${ }^{15}$, Seema Irfan ${ }^{16}$, Stephen Baker, ${ }^{9,10}$, Steve P.

8 Luby $^{1}$, Yanjia Cao ${ }^{1}$, Zoe Anne Dyson ${ }^{10,17,18,19}$, Denise O. Garrett ${ }^{12}$, Jacob John ${ }^{20}$, Gagandeep

$9 \mathrm{Kang}^{21}$, Yogesh Hooda ${ }^{2,22}$, Samir K. Saha ${ }^{2,23},{ }^{\dagger}$ Senjuti Saha ${ }^{2}{ }^{\dagger} \mathrm{Jason} \mathrm{R}$. Andrews $^{1 \dagger}$

$11{ }^{1}$ Division of Infectious Diseases and Geographic Medicine, Stanford University, Stanford, CA,

12 USA.

$13 \quad{ }^{2}$ Child Health Research Foundation, Dhaka, Bangladesh.

$14{ }^{3}$ Department of Medical Microbiology and Infectious Diseases, Erasmus University Medical

15 Center, Rotterdam, The Netherlands.

$16{ }^{4}$ Department of Clinical Microbiology, Christian Medical College, Vellore, India.

$17{ }^{5}$ Department of Pediatrics and Child Health, Aga Khan University, Karachi, Pakistan.

$18{ }^{6}$ Institute of Biodiversity, Animal Health and Comparative Medicine, University of Glasgow,

19 Glasgow, UK.

$20{ }^{7}$ Cambridge Institute of Therapeutic Immunology \& Infectious Disease (CITIID) Department of

21 Medicine, University of Cambridge, Cambridge, UK.

$22{ }^{8}$ Department of Community Medicine, Kathmandu University School of Medical Sciences, Nepal. 
$23{ }^{9}$ University of Cambridge School of Clinical Medicine, Cambridge Biomedical Campus,

24 Cambridge, UK.

$25{ }^{10}$ Department of Medicine, University of Cambridge School of Clinical Medicine, Cambridge

26 Biomedical Campus, Cambridge, UK.

$27{ }^{11}$ Department of Medicine, University of Toronto, Toronto, Canada.

$28 \quad{ }^{12}$ Applied Epidemiology, Sabin Vaccine Institute, Washington, DC, USA.

$29{ }^{13}$ Dhulikhel Hospital, Kathmandu University Hospital, Kavrepalanchok, Nepal.

$30{ }^{14}$ Central Department of Microbiology at Tribhuvan University, Kirtpur, Nepal.

$31{ }^{15}$ Department of Pharmacology, Kathmandu University School of Medical Sciences, Dhulikhel,

32 Nepal.

$33{ }^{16}$ Department of Pathology and Microbiology, Aga Khan University Hospital, Karachi, Pakistan.

$34 \quad{ }^{17}$ London School of Hygiene \& Tropical Medicine, London, UK.

$35{ }^{18}$ Department of Infectious Diseases, Central Clinical School, Monash University, Melbourne,

36 Victoria, Australia.

$37{ }^{19}$ Wellcome Sanger Institute, Wellcome Genome Campus, Hinxton, Cambridge, UK.

$38{ }^{20}$ Department of Community Health, Christian Medical College, Vellore, India.

$39{ }^{21}$ The Wellcome Trust Research Laboratory, Division of Gastrointestinal Sciences, Christian

40 Medical College, Vellore, India.

$41 \quad{ }^{22}$ MRC Laboratory Molecular Biology, Cambridge, UK.

$42{ }^{23}$ Department of Microbiology, Dhaka Shishu Hospital, Dhaka, Bangladesh.

$43 *$ Contributed Equally

$44+$ Contributed Equally 
medRxiv preprint doi: https://doi.org/10.1101/2021.09.03.21262852; this version posted September 7, 2021. The copyright holder for this preprint

(which was not certified by peer review) is the author/funder, who has granted medRxiv a license to display the preprint in perpetuity.

It is made available under a CC-BY-NC-ND 4.0 International license .

46 Word Count (intro, results, discussion): 2,913

47 Abstract Word Count: 109

48 Figures: 5

49 Tables: 0

50

51 Correspondence:

52 Jason R. Andrews

53 Division of Infectious Diseases and Geographic Medicine

54 Stanford University School of Medicine

55 Biomedical Innovations Building, Room 3458

56 Stanford, CA 94025, USA

57 Email: jandr@stanford.edu

58 Phone: +1 6504972679

59 


\section{Abstract}

61

62 The emergence of increasingly antimicrobial-resistant (AMR) Salmonella enterica serovar Typhi

63 (S. Typhi) threatens to undermine effective treatment and control. Here, aiming to investigate the

64 temporal and geographic patterns of emergence and spread of AMR S. Typhi, we sequenced

$653,489 S$. Typhi isolated from prospective surveillance in South Asia and combined these with a

66 global collection of 4,169S. Typhi genomes. Our analysis revealed that independent acquisition

67 of plasmids and homoplastic mutations conferring AMR have occurred repeatedly in multiple

68 lineages of $S$. Typhi, predominantly arising in South Asia. We found evidence of frequent

69 international and intercontinental transfers of AMR $S$. Typhi, followed by rapid expansion and

70 replacement of antimicrobial-susceptible clades. 


\section{Introduction}

74 Typhoid fever, the disease caused by Salmonella enterica serovar Typhi (S. Typhi), remains a

75 major worldwide public health concern (1). The organism causes 11 million illnesses and

$76>100,000$ deaths annually $(2,3)$. The highest typhoid fever incidence rates occur in South Asia,

77 which contains $70 \%$ of the global disease burden, but substantial morbidity and mortality also

78 occur in sub-Saharan Africa, Southeast Asia, and Oceania. In the pre-antimicrobial era, the case

79 fatality rate of typhoid fever was around $15 \%$, but morbidity and mortality declined dramatically

80 following the introduction of chloramphenicol in the 1940s, followed by ampicillin and

81 trimethoprim-sulfamethoxazole in the 1960s $(4,5)$.

83 The effectiveness of antimicrobial therapy for typhoid fever has been repeatedly threatened by

84 the emergence and expansion of organisms that exhibit resistance to the principal antimicrobials.

85 Multi-drug resistant (MDR) variants, harboring genes encoding resistance to ampicillin,

86 chloramphenicol, and trimethoprim-sulfamethoxazole first emerged in the 1970s; subsequently,

87 a single lineage (H58; 4.3.1) was introduced into sub-Saharan Africa and Southeast Asia from

88 South Asia and became globally dominant (6,7). The genes facilitating resistance to these

89 specific antimicrobials were originally only located on IncHI1 plasmids but later also became

90 integrated into the chromosome. The fluoroquinolones were initially highly effective against

91 early MDR $S$. Typhi organisms and became the mainstay of therapy in South Asia in the 1990s.

92 However, fluoroquinolone non-susceptible isolates started to emerge in the mid 1990s and,

93 within 20 years, the majority of $S$. Typhi in South Asia contained mutations in the quinolone

94 resistance-determining regions (QRDR) (4) (8). In 2016, a large outbreak of $S$. Typhi containing

95 plasmid-mediated resistance to third generation cephalosporins and fluoroquinolones and 
96 chromosomally located genes encoding the MDR phenotype was identified in Pakistan and

97 termed extensively drug-resistant (XDR) (9). More recently, a single polymorphism in the AcrB

98 efflux pump conferring resistance to azithromycin was found to have independently arisen in

99 multiple lineages of $S$. Typhi, threatening the efficacy of all oral antibiotic antimicrobials for

100 typhoid treatment (10). Taken together, $S$. Typhi has exhibited resistance to all oral drugs known

101 to be effective for its treatment, although it has not yet been identified in the same clone.

102

103 Typhoid conjugate vaccines (TCV) have recently proven effective for disease prevention, and the

104 World Health Organization recommends prioritized introduction in countries with a high burden

105 of antimicrobial resistant $S$. Typhi (11). However, given the current trajectory of antimicrobial

106 resistant (AMR) in $S$. Typhi waiting until a high AMR burden is present within a country to

107 introduce typhoid vaccines may ill-advised. Understanding the historical emergence, expansion

108 and geographic spread of antimicrobial resistant $S$. Typhi may yield insights into where resistant

109 strains might spread and how quickly they will become dominant within populations.

111 Here, we leveraged prospective, population-based typhoid surveillance studies from four of the

112 highest burden countries in South Asia: Bangladesh, India, Nepal and Pakistan. We sequenced

$1133,489 S$. Typhi organisms isolated over a six-year period, and these data were combined with a

114 global collection of $>4,000$ additional genomes sequences to investigate the emergence and

115 geographical spread of AMR S. Typhi over the past three decades.

\section{Results}

118 Genotypic diversity and phylogenetic structure of S. Typhi in South Asia 
119 A total of 3,489 S. Typhi isolates from four countries (Bangladesh, India, Nepal, and Pakistan)

120 collected between 2014 and 2019 were sequenced. Genotype analysis identified 29 distinct

121 genotypes (Figure S1). The majority of isolates $(2,474 ; 70.9 \%)$ belonged to genotype 4.3 .1

122 (haplotype H58). The major sublineages of H58 (lineage I and lineage II) were common, with

123 H58 lineage I (genotype 4.3.1.1) dominant in Bangladesh and Pakistan, and H58 lineage II

124 (genotype 4.3.1.2) the most common genotype in India and Nepal. Among non-H58 isolates, the 125 most common subclades were subclade $3.2 .2(190 ; 5.5 \%), 3.3 .2(161 ; 4.6 \%), 2.3 .3(140 ; 4.0 \%)$, $126 \quad 2.5(123 ; 3.5 \%)$, and $3.3 .1(85 ; 2.4 \%)$.

128 We identified multiple, phylogenetically linked sub-lineages shared across South Asia (Figure

129 S2), most regularly between Bangladesh, Nepal, and India. Within the H58 isolates, S. Typhi

130 4.3.1.2 isolates formed distinct clades with intermingled isolates from India and Nepal, while 131 4.3.1.3 isolates, identified predominantly in Bangladesh, clustered with few isolates from India. In 132 contrast, the H58 isolates from Pakistan largely clustered independently and was dominated by a 133 monophyletic XDR clade (4.3.1.1.P1).

136 To provide additional context for the 3,489 S. Typhi new South Asian genome sequences, and 137 better understand temporal and spatial distribution of lineages and antimicrobial resistance, we 138 constructed a further phylogeny incorporating an additional 4,169 S. Typhi sequences from 139 organisms isolated from 1905 to 2018 from $>70$ countries (Figure 1). Overall, the new 140 sequences clustered with previously sequenced South Asian isolates, generating distinct 141 geographic structure. Genotype 4.3.1 formed a large subclade. Primary clades 2, 3 and 4 were 
142 distributed across continents with limited isolates outside these clades. Notably, four subclades

$143(2.3 .3,2.5,3.2 .2$, and 3.3) were dominant in South Asia, accounting for $75.7 \%$ of all non-H58

144 organisms.

146 We classified isolates as multidrug-resistant if they containing genes conferring resistance to

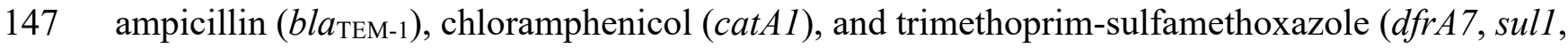

148 or sul2). From 2000 onwards, we observed a declining trend in MDR isolates in Bangladesh and

149 India, a stable low proportion $(<5 \%)$ in Nepal, and an increasing proportion in Pakistan and

150 Africa (Figure S3a). Acquired resistance genes that contribute to the MDR phenotype were

151 identified in $26.8 \%$ of the isolates of the global genome collection, including $98.4 \%$ of the H58

152 isolates, in comparison to just $1.6 \%$ of the non-H58 isolates $(1.1 .1,2.5 .1,3.1 .1,3.2 .1,3.4,4)$.

153 Among these rare non-H58 multidrug-resistant isolates, resistance was almost exclusively

154 entirely plasmid-mediated (96.9\%). In contrast, for H58 isolates we observed that plasmid-

155 mediated resistance was persistent in the H58 isolates in the 1990s, but from 2000 onward was

156 less frequent, with most MDR isolates containing chromosomal insertions of drug-resistance

157 genes $(75.2 \%)$.

159 In contrast to the temporal trends in MDR, there was a consistent rise in the proportion of global

$160 S$. Typhi that were fluoroquinolone non-susceptible (FQNS), primarily associated with mutations

161 gryA, gyrB, parC, and parE) (Figure S3b). The most dramatic increase in FQNS S. Typhi

162 occurred in Bangladesh, exceeding $85 \%$ by early 2000 , followed by India, Pakistan, and Nepal,

163 reaching $>95 \%$ in all four South Asian countries. FQNS $S$. Typhi increased from $<20 \%$ in 2007

164 year to $>60 \%$ by 2011 in Southeast Asia. In Africa, this increase occurred more recently, 
165 originating in 2010. Overall, we found that QRDR mutations were significantly more common in

166 the H58 isolates (89.1\%) compared with other lineages $(45.4 \%)(p<0.0001)$. From 2010

167 onwards, an increasing number of isolates containing multiple QRDR mutations; $>10 \%$ of all

168 isolates harbored three mutations (Figure S3). Among the novel genome sequences, 437 were

169 'triple mutants' (Table S3), which are highly associated with full resistance and failure to

170 respond to fluoroquinolone therapy (12). The majority (402/437; 92\%) of these organisms

171 occurred in H58 lineage II (4.3.1.2) in India and Nepal; the second most common (15/437; 3.4\%)

172 triple mutant genotype was 3.3 and predominantly isolated in India.

173

174 Susceptibility to fluoroquinolones can be further reduced via plasmid-mediated acquisition of

$175 q n r$ genes. We identified qnrS in two non-H58 isolates (genotype 3) and 686 H58 isolates that

176 included genotype 4.3.1 $(\mathrm{n}=3), 4.3 .1 .1(\mathrm{n}=5)$, 4.3.1.P1 $(\mathrm{n}=550)$, and 4.3.1.3 $(\mathrm{n}=125)$. Most

177 H58 lineage I isolates from Pakistan were XDR (4.3.1.P1) carrying the previously identified

178 composite transposon containing bla $\mathrm{TEM}-1_{1}, \mathrm{catA1}$, dfrA7, sull, sul2 inserted in the chromosome,

179 and bla $a_{\mathrm{CTX}-\mathrm{M}-15}$ and $q n r S$ associated with an IncY plasmid (9). Azithromycin resistance, conferred

180 by $a c r B$ mutations (R717Q and R717L), was identified in 54 isolates across eight different

181 genotypes including genotype 4.3.1 $(\mathrm{n}=1), 4.3 .1 .1(\mathrm{n}=31), 4.3 .1 .2(\mathrm{n}=5), 4.3 .1 .3(\mathrm{n}=2)$, and

182 non-H58 isolates comprising, genotype 2.3.3 $(\mathrm{n}=2), 3.2 .2(\mathrm{n}=9), 3.3 .2(\mathrm{n}=3)$ and 3.5.4 $(\mathrm{n}=$

183 1).

184

185 Antimicrobial resistance and growth of the effective population size

186 To investigate how the AMR has shaped the effective population size of $S$. Typhi, we generated

187 timed phylogenies and modeled the effective population size of antimicrobial susceptible and 
192 2017, while triple mutants have steadily increased from 2010 (Figure 2). In Pakistan, the $\mathrm{N}_{\mathrm{e}}$ of

193 non-XDR H58 S. Typhi increased from 2000 until around 2015 and began to fall; XDR

194 organisms emerged and have been rapidly growing in frequency since 2016, eclipsing the

195 effective population of non-XDR organisms by 2018. In Bangladesh, the $\mathrm{N}_{\mathrm{e}}$ of $\mathrm{H} 58$ had slowly

196 declined from around 2010; however, azithromycin-resistant organisms emerged in 2013 with a

197 corresponding increase in $\mathrm{N}_{\mathrm{e}}$. In all three settings, organisms with key AMR conferring

198 mutations or genes appear to be replacing their susceptible (or, in the case of fluoroquinolones,

199 less-resistant) counterparts.

202 Using country of sampling as a discrete trait, we generated dated phylogenies to reconstruct the 203 evolutionary history and geographic spread of H58 lineage and the four 4 common non-H58

204 genotypes. These five genotypes accounted for $75 \%$ of all isolates from the past decade. The 205 mean nucleotide substitution rates for each lineage are described in Table $\mathbf{S 4}$.

207 Phylogeographic reconstruction of H58 isolates (4.3.1, 4.3.1.1, 4.3.1.1.P1, 4.3.2.1 and 4.3.1.3)

208 estimated that the time of most recent common ancestor (tMRCA) of all contemporary H58 $S$.

209 Typhi strains existed around 37 years ago (1984). The distribution of isolates and the tree 210 topology are consistent with at least 138 international transfer events, including multiple 
211 introductions within South Asia and dissemination from South Asia into Southeast Asia and

212 Africa, as well as many travel-related cases identified in the U.K. and U.S. (Figure 3, Figure 4).

213 The distribution of QRDR mutations within the phylogeny demonstrated that these resistance-

214 conferring mutations have arisen independently on at least 80 distinct occasions. We also

215 predicted that ciprofloxacin-resistant triple mutant isolates most likely originated in India around

2161996 and were introduced into Pakistan between 2005 and 2013 and into Nepal on at least three

217 occasions (2003-2015). Furthermore, we identified frequent transmissions of international

218 transfer of MDR isolates $(\mathrm{n}=33)$, with multiple introductions from South Asia followed by local

219 expansion. In addition, the phylogeographic analyses also showed that the Pakistani XDR

220 lineage (genotype 4.3.1.1.P1) emerged around 2015 and has been subsequently identified on

221 multiple occasions in United Kingdom and United States.

222

223 The major non-H58 clades also acquired AMR loci and spread within and from South Asia.

224 Genotype 2.3.3 circulated predominantly in Bangladesh but spread to Pakistan and India within

225 the past 30 years (Figure S5). QRDR mutations within genotype 2.3.3 have emerged

226 independently on at least three occasions. Genotype 2.5, which may have circulated in India for

$227>100$ years (Figure S6), has been transferred to sub-Saharan Africa and Nepal multiple times,

228 including two instances with strains containing QRDR mutations since 2015. Genotype 3.2.2

229 organisms originating from Bangladesh were observed in South Asia only. We observed a single

230 instance of transfer from Bangladesh to Nepal and ongoing local expansion. In contrast, we

231 found that these organisms have been regularly transferred from Bangladesh to India (Figure

232 S7). Transfer events included at least four recent introductions of FQNS organisms between

2332006 and 2017. The most recent common ancestor of genotype 3.3 was estimated to have been 
234 from India >200 years ago (Figure S8), but moved extensively across South Asia, establishing 235 large subclades in Bangladesh and Nepal, before progressing to sub-Saharan Africa, the Middle

236 East, and Southeast Asia. Genotype 3.3 organisms with QRDR mutations have moved from India

237 to Nepal on multiple occasions.

239 Overall, our analysis identified evidence for 197 introduction events between countries, of which

240138 were intracontinental and 59 were intercontinental (Figure 5). The most common

241 international transmission events were within South Asia and from South Asia to Southeast Asia,

242 East and Southern Africa. We estimated that resistance-conferring mutations to fluoroquinolones

243 or azithromycin have independently emerged on at least 101 separate occasions within the last 30

244 years, mostly in South Asian countries $(n=94)$, and occasionally arising in Southeast Asia,

245 Africa and South America. In addition, isolates carrying QRDR mutations were recently

246 transferred between countries on at least 119 independent occasions.

\section{Discussion}

249 This analysis of $S$. Typhi genome sequences reveals that the acquisition through plasmid

250 acquisition or frequent, homoplastic mutations occurring across multiple lineages, has been

251 accompanied by expansion and international spread of AMR $S$. Typhi clones. We identified

252 numerous international and intercontinental transfers of $S$. Typhi over the past thirty years, with

253 the majority associated with AMR phenotypes. Once introduced to a new setting, AMR $S$. Typhi

254 become quickly fixed, as broadly exemplified with fluoroquinolone non-susceptible clades in

255 multiple countries and XDR S. Typhi in Pakistan. This rapid emergence, spread and fixation of

256 AMR in $S$. Typhi suggests that making decisions regarding TCV introduction via current AMR 
259 regularly move internationally, underscoring the need for resources to support typhoid control in

260 this region.

262 Our data are consistent with recent studies suggesting that MDR $S$. Typhi-strains resistant to

263 the classical first line drugs - is now generally on the decline in South Asia $(13,14)$. The decline

264 of MDR $S$. Typhi in Asia has been accompanied by a decrease in the proportion of isolates

265 carrying IncHI1 plasmids (except for Pakistan, where the MDR decline abruptly reversed amid

266 the emergence of the XDR lineage). In our study, MDR was principally associated with H58

267 carrying chromosomally integrated AMR genes. The integration of AMR genes into the $S$. Typhi

268 chromosome remains a concern, as it provides a mechanism for stable vertical transmission of

269 MDR phenotypes $(6,15)$. In contrast to South Asia, MDR typhoid associated with H58 and non-

$270 \mathrm{H} 58$ isolates appears to be increasing in parts of Africa, with outbreaks being reported in the last

271 decade $(16,17)$.

273 QRDR mutations have independently arisen in all $S$. Typhi lineages due to sustained

274 fluoroquinolone exposure. Nearly all of organisms containing QRDR mutations appear to have

275 arisen in South Asia, and many have spread regionally and globally. Notably, our analysis

276 revealed that highly fluoroquinolone resistant $S$. Typhi triple mutants have recently emerged in

277 six different genotypes, including H58 lineage (4.3.1), lineage I (4.3.1.1), lineage II (4.3.1.2),

278 and non-H58 isolates (3.3). Our phylogeography analysis suggests that these isolates most likely

279 originated in India and disseminated to neighboring countries including Nepal and Pakistan. 
281 The recent emergence and spread of resistance to third-generation cephalosporins and

282 azithromycin further complicates typhoid fever treatment $(8,9)$. Within three years of its first

283 recognition, the XDR genotype (4.3.1.1.P1) became the dominant genotype circulating in

284 Pakistan. The same sub-clade has been isolated from travelers returning from Pakistan to

285 Canada, United Kingdom and United States $(18,19)$. Recently, there have been reports of non-

286 travel associated cases of 4.3.1.1.P1 XDR $S$. Typhi in the United States, suggestive of local

287 transmission following importation (19). The XDR genotype has been typically associated with a

288 composite MDR transposon inserted in the chromosome and by the acquisition of an IncY

289 plasmid carrying bla $a_{\mathrm{CTX}-\mathrm{M}-15}$ and $q n r S 1$ (9). However, a recent study reported multiple

290 integrations of bla ${ }_{\mathrm{CTX}-\mathrm{M}-15}$ from the IncY plasmid into the chromosome of XDR $S$. Typhi isolates

291 (20). Chromosomal integration of bla $a_{\mathrm{CTX}-\mathrm{M}-15}$ may lessen its impact fitness cost, making it more

292 likely that resistance will be maintained even in the absence of selection pressure in a

293 comparable manner to the MDR gene cassette integration $(15,20)$.

295 At present, all XDR $S$. Typhi strains identified have been susceptible to azithromycin and

296 carbapenems (19). Concerningly, azithromycin-resistant $S$. Typhi have recently been reported in

297 Bangladesh, India, Pakistan, Nepal and Singapore (10,21,22), arising from mutations in $a c r B$.

298 These mutations have arisen independently multiple times in distinct lineages (10). To date,

299 XDR $S$. Typhi isolates containing mutations in $a c r B$ have not yet been identified. Such

300 organisms would preclude effective treatment with established oral antimicrobials, which could

301 lead to increased hospitalization rates, greater morbidity and mortality. 
303 Our findings should be interpreted within the context of the limitations of the available data.

304 While this analysis included the largest collection of novel $S$. Typhi genome sequences to date,

305 there remains underrepresentation of sequences from several regions, including

306 disproportionately small numbers from many countries in sub-Saharan Africa and Oceania where

307 typhoid is endemic. Even in countries with more dense sampling, most isolates were derived

308 from a small number of surveillance sites and may not be representative of the distribution of

309 circulating strains. Because $S$. Typhi genomes only cover a fraction of all typhoid fever cases,

310 phylogenies are highly incomplete; our estimates for AMR-conferring homoplastic mutations

311 and international transfers represent lower bounds like may substantially underestimate their true

312 frequency. These circumstances highlight the need for expanding genomic surveillance for

313 enteric pathogens to provide a more comprehensive window into the emergence, expansion and

314 spread of antimicrobial-resistant organisms.

316 The present study highlights the sustained emergence and geographic spread of AMR S. Typhi

317 strains, with evidence of frequent international exportation. This observation underscores the

318 importance of approaching typhoid fever control and antimicrobial resistance as a global rather

319 than local problem. The recent emergence of XDR and azithromycin-resistant $S$. Typhi creates

320 greater urgency for rapidly expanding prevention measures, including use of TCV and

321 improvements to water and sanitation infrastructure, in typhoid-endemic countries. Such

322 measures are needed in countries where AMR prevalence among $S$. Typhi isolates is currently

323 high, but given the propensity for international spread, should not be restricted to such setting.

\section{Methods}


medRxiv preprint doi: https://doi.org/10.1101/2021.09.03.21262852; this version posted September 7, 2021. The copyright holder for this preprint (which was not certified by peer review) is the author/funder, who has granted medRxiv a license to display the preprint in perpetuity.

It is made available under a CC-BY-NC-ND 4.0 International license .

Bacterial isolates

327 This study included $S$. Typhi isolates obtained from the Surveillance for Enteric Fever in Asia

328 Project (SEAP; Bangladesh, Nepal, and Pakistan; 2016-2019), Etiologies of Acute Febrile Illness

329 Study (Nepal; 2014-2016), and Surveillance for Enteric Fever in India Project (SEFI; 2017-2019.

330 The study methodologies have been previously described (23-25). In brief, participants for these

331 studies included individuals of all ages presenting to study site facilities with febrile illness.

332 These included 5 facilities in Dhaka, Bangladesh, 18 facilities across 16 cities in India, 11

333 facilities across 3 cities in Nepal and two hospitals and a university laboratory network in

334 Karachi, Pakistan. Across these studies, there were a total of 9,945 blood culture-confirmed

335 typhoid cases. From these, we selected a country-stratified sample of 3,489 $S$. Typhi isolates for

336 sequencing. Details on the sampling for each country are available in the Appendix.

338 Whole-genome sequencing

339 Whole-genome sequencing (WGS) was performed at the Wellcome Trust Sanger Institute using

340 the Illumina Hiseq2500 platform (Illumina, San Diego, CA, USA) to generate paired-end reads

341 of 100-150 bp in length, and at a commercial service in Bangalore and at the Wellcome Trust

342 Research Laboratory in the Christian Medical College using the Illumina Miseq platform

343 (Illumina, San Diego, CA, USA). Sequence data quality was checked using FastQC v0.11.9 to

344 remove low quality reads (26). We summarized all quality indicators using MultiQC v1.7 (27).

345 Species identification was confirmed with Kraken2 (28), and the Salmonella in silico Typing

346 Resource (SISTR) was used for WGS-based serotyping (29). Short Read Sequence Typing for

347 Bacterial Pathogens (SRST2) (30) was used to map known alleles and identify MLSTs directly 
348 from reads according to the Salmonella enterica MLST scheme

349 (https://pubmlst.org/salmonella/).

Mapping and SNP analysis

352 Paired-end Illumina reads were mapped to the $S$. Typhi CT18 (accession no. AL513382)

353 reference chromosome sequence using RedDog mapping pipeline v1beta.11 (https://github.com/

354 katholt/reddog). RedDog uses Bowtie2 v2.4.1 (31) to map reads to the reference genome, and

355 SAMtools v1.10 (32) to identify SNPs that have a phred quality score above 30, and to filter out

356 those SNPs supported by less than five reads, or with $2.5 \mathrm{x}$ the average read depth that represent

357 putative repeated sequences, or those that have ambiguous base calls. For each SNP that passes

358 these criteria in any one isolate, consensus base calls for the SNP locus were extracted from all

359 genomes mapped, with those having phred quality scores under 20 being treated as unknown

360 alleles and represented with a gap character.

362 Chromosomal SNPs with confident homozygous calls (phred score above 20 ) in $>95 \%$ of the

363 genomes mapped (representing a 'soft' core genome) were concatenated to form an alignment of

364 alleles using the RedDog python script parseSNPtable.py with parameters -m cons, aln and -c

3650.95 and SNPs called in prophage regions and repetitive sequences (354 kb; 7.4\% of bases) in

366 the CT18 reference chromosome, as defined previously (33) were excluded to form an alignment

367 of 14,901 variant sites. SNPs occurring in recombinant regions were detected by Gubbins v2.4.1

368 (34) and excluded resulting in a final alignment of 11,978 chromosomal SNPs. The SNP data

369 were used to assign all isolates to previously defined genotypes according to an extended $S$.

370 Typhi genotyping framework using the GenoTyphi python script 
371 (https://github.com/katholt/genotyphi) (33). To provide global context, additional $S$. Typhi

372 genomes $(9,12,17,33,35-41)$ were subjected to both SNP calling, recombination filtering, and

373 genotyping as described above, resulting in an alignment of 28,897chromosomal SNPs.

375 Phylogenetic analyses

376 Maximum likelihood (ML) phylogenetic trees were inferred from the chromosomal SNP

377 alignments using RAxML v8.2.10 (42) (command raxmlHPC-PTHREADS). A generalized time-

378 reversible model and a Gamma distribution was used to model site-specific rate variation (GTR+

$379 \Gamma$ substitution model; GTRGAMMA in RAxML) with 100 bootstrap pseudoreplicates used to

380 assess branch support for the ML phylogeny. We selected the single tree with the highest

381 likelihood score as the best tree. The resulting phylogenies were visualized and annotated using

382 the iTOL v5 online version (43).

Temporal and phylogeographic analysis

385 To investigate dates of emergence and geographical transfers, we inferred timed phylogenies

386 using globally and temporally representative samples. First, we used TempEst v1.5 to assess

387 temporal structure by conducting a regression of the root-to-tip branch distances of the tree as a

388 function of the sampling time (44), which was confirmed by a clustered permutation test using

389 BactDating (45). For the non-H58 isolates, we estimated the best-fit models, tree topology,

390 evolutionary rates, and phylogeography by using a Bayesian Markov chain Monte Carlo

391 (MCMC) method with the software package BEAST2 v2.6.2 (46). Separate trees were fit for

392 each of the most common non-H58 lineages (2.3.3, 2.5, 3.2.2, and 3.3). Isolates from each 
medRxiv preprint doi: https://doi.org/10.1101/2021.09.03.21262852; this version posted September 7, 2021. The copyright holder for this preprint (which was not certified by peer review) is the author/funder, who has granted medRxiv a license to display the preprint in perpetuity.

It is made available under a CC-BY-NC-ND 4.0 International license .

393 lineage were selected based on temporal, geographic and phylogenetic diversity, as described in

394 detail in the Appendix.

395

396 For the BEAST analysis, a GTR $+\Gamma$ substitution model was selected, and the sampling times (tip

397 dates) were defined as the year of isolation to calibrate the molecular clock. We tested support

398 for a strict clock for each lineage using the relaxed clock test in treedater, and the strict clock

399 was rejected in each instance (47). We therefore constructed time-phylogenies using coalescent

400 exponential population priors with a relaxed clock (uncorrelated lognormal distribution) $(6,17)$.

401 Three independent runs were performed to ensure convergence, and were combined with

402 LogCombiner, following removal of the first 10 million steps from each as burn-in. The effective

403 sample sizes (ESSs) of the parameters were estimated to be $>200$ for all independent runs of the

404 analysis. The trees were summarized in a maximum clade credibility (MCC) target tree using the

405 Tree Annotator program v2.4.7. The time of the most recent common ancestor (tMRCA)

406 estimates were calculated as the years before the most recent sampling dates. Phylogeographical

407 reconstruction was obtained by the continuous-time Markov Chain process over discrete

408 sampling locations implemented in BEAST. The final trees were visualized using FigTree v1.4

409 (http://tree.bio.ed.ac.uk/software). For H58, we had 4,761 isolates, which precluded temporal and

410 phylogeographic analysis using BEAST due to computational constraints. To avoid significant

411 down-sampling of isolates, we used the treedater $\mathrm{R}$ package (47) with an uncorrelated, relaxed

412 molecular lock to estimate the timed phylogeny for H58, which yielded a tMRCA matching a

413 root-to-tip based analysis using BactDating and consistent with previously published estimates

414 (6). We included all H58 sequences available in our collection to improve accuracy of the timed

415 phylogeny and phylogeographic analysis. We reconstructed the ancestral state of nodes using the 
416 maximum parsimony approach with the Phangorn R package, considering important events with

417 a location probability of $>0.5$ between connected nodes. For visualization purposes, we selected

418 a smaller subset of sequences to depict in a dated phylogenetic tree. For all phylogeographic

419 analyses, we considered a geographic transfer when the most probable location between two

420 connected nodes (or between a node and a tip) differed, and we considered the time window of

421 transfer as the date range between the nodes (or between the node and tip). The geospatial

422 transmissions of lineage strains from the phylogeographic reconstructions were analyzed and

423 visualized using ArcMap 10.7.1 (https://desktop.arcgis.com/en/arcmap/).

426 To evaluate the historical effective population size for H58 lineage strains, we used the time-

427 stamped H58 tree to estimate the effective population size through time using

428 the skygrowth package (https://github.com/mrc-ide/skygrowth). We pruned the timed

429 phylogenies by country and antimicrobial resistance pattern to compare the effective population

430 sizes of antimicrobial resistant and sensitive populations.

432 AMR associated genes detection and plasmid replicon analysis

433 ARIBA (Antimicrobial Resistance Identifier by Assembly) v2.10.0 and CARD database v1.1.8

434 (https://card.mcmaster.ca/home) were used to investigate antimicrobial resistance gene content.

435 Point mutations in the quinolone resistance determining region (QRDR) of the DNA-gyrase

$436 \mathrm{gyr} A / B$ and topoisomerase-IV $\operatorname{par} C / E$ genes, associated with reduced susceptibility to

437 fluoroquinolones and quinolone resistance genes $(q n r S)$ were also detected using ARIBA.

438 Isolates were defined as being MDR if resistance genes were detected by ARIBA in the beta- 
439 lactam, trimethoprim/sulphonamide and chloramphenicol classes. Plasmid replicons were

440 identified using ARIBA and the PlasmidFinder database (30).

442 Data availability

443 Illumina sequence data was submitted to the European Nucleotide Archive. Sequence data from

$4444,169 S$. Typhi strains from previous studies were also included for global context raw sequence

445 data for these isolates are available in European Nucleotide Archive. Details and individual

446 accession numbers of sequence data included in our analysis have been included in Tables S1

447 and S2.

449 Ethics statement

450 Ethical approval for the parent studies were obtained from the Bangladesh Institute of Child

451 Health Ethical Review Committee, Christian Medical College Institutional Review Board, Nepal

452 Health Research Council, Aga Khan University Hospital Ethics Committee and Pakistan

453 National Ethics Committee, Stanford University Institutional Review Board, and U.S. Centers

454 for Disease Control and Prevention. Informed written consent and clinical information were

455 taken from adult participants and legal guardians of child participants.

457 Acknowledgements

458 This work was supported by a grant from the Bill and Melinda Gates Foundation (grant number $459 \quad$ INV-008335). 


\section{References}

463 1. Dougan G, Baker S. Salmonella enterica Serovar Typhi and the Pathogenesis of Typhoid Fever . Annu Rev Microbiol. 2014;

465 2. Antillón M, Warren JL, Crawford FW, Weinberger DM, Kürüm E, Pak GD, et al. The burden of typhoid fever in low- and middle-income countries: A meta-regression approach. PLoS Negl Trop Dis. 2017;

3. Stanaway JD, Reiner RC, Blacker BF, Goldberg EM, Khalil IA, Troeger CE, et al. The global burden of typhoid and paratyphoid fevers: a systematic analysis for the Global Burden of Disease Study 2017. Lancet Infect Dis. 2019;19(4):369-81.

4. Andrews JR, Qamar FN, Charles RC, Ryan ET. Extensively Drug-Resistant Typhoid Are Conjugate Vaccines Arriving Just in Time? N Engl J Med [Internet]. 2018 Oct 12;379(16):1493-5. Available from: https://doi.org/10.1056/NEJMp1803926

5. Mogasale V, Maskery B, Ochiai RL, Lee JS, Mogasale V V., Ramani E, et al. Burden of

6. Wong VK, Baker S, Pickard DJ, Parkhill J, Page AJ, Feasey NA, et al. Phylogeographical analysis of the dominant multidrug-resistant H58 clade of Salmonella Typhi identifies inter-and intracontinental transmission events. Nat Genet. 2015;

7. Dyson ZA, Klemm EJ, Palmer S, Dougan G. Antibiotic resistance and typhoid. Clin Infect Dis. 2019;

8. Hooda Y, Sajib MSI, Rahman H, Luby SP, Bondy-Denomy J, Santosham M, et al. Molecular mechanism of azithromycin resistance among typhoidal Salmonella strains in Bangladesh identified through passive pediatric surveillance. PLoS Negl Trop Dis. 2019;

9. Klemm EJ, Shakoor S, Page AJ, Qamar FN, Judge K, Saeed DK, et al. Emergence of an

10. Sajib MSI, Tanmoy AM, Hooda Y, Rahman H, Andrews JR, Garrett DO, et al. Tracking the emergence of azithromycin resistance in multiple genotypes of typhoidal salmonella. MBio. 2021;12(1):1-12.

11. World Health Organization. Typhoid vaccines: WHO position paper, March 2018 - 
Recommendations. Vaccine [Internet]. 2019;37(2):214-6. Available from: https:/www.sciencedirect.com/science/article/pii/S0264410X18304912

12. Thanh DP, Karkey A, Dongol S, Thi NH, Thompson CN, Rabaa MA, et al. A novel ciprofloxacin-resistant subclade of h58. Salmonella typhi is associated with fluoroquinolone treatment failure. Elife. 2016;

13. Carter AS, Luby SP, Garrett DO. Introducing Typhoid Conjugate Vaccine in South Asia: [Internet]. 2020 Dec 1;71(Suppl 3):S191-5. Available from:

14. Balaji V, Kapil A, Shastri J, Pragasam AK, Gole G, Choudhari S, et al. Longitudinal Typhoid Fever Trends in India from 2000 to 2015. Am J Trop Med Hyg [Internet]. 2018/07/24. 2018 Sep;99(3_Suppl):34-40. Available from: https://pubmed.ncbi.nlm.nih.gov/30047367

15. Holt KE, Baker S, Weill FX, Holmes EC, Kitchen A, Yu J, et al. Shigella sonnei genome sequencing and phylogenetic analysis indicate recent global dissemination from Europe. Nat Genet. 2012;44(9):1056-9.

16. Consortium IT, Wong VK, Holt KE, Okoro C, Baker S, Pickard DJ, et al. Molecular Surveillance Identifies Multiple Transmissions of Typhoid in West Africa. PLoS Negl

17. Park SE, Pham DT, Boinett C, Wong VK, Pak GD, Panzner U, et al. The phylogeography Trop Dis [Internet]. 2016 Sep 22;10(9):e0004781-e0004781. Available from:

516 18. Eshaghi A, Zittermann S, Bharat A, Mulvey MR, Allen VG, Patel SN. Importation of Extensively Drug-Resistant \&lt;span class=\&quot;named-content genus-species\&quot; id=\&quot;named-content-1\&quot;\&gt;Salmonella enterica\&lt;/span\&gt; Serovar Typhi Cases in Ontario, Canada. Antimicrob Agents Chemother [Internet]. 2020 Apr 21;64(5):e02581-19. Available from: http://aac.asm.org/content/64/5/e02581-19.abstract

19. Health Alert Network C. Extensively Drug-Resistant Salmonella Typhi Infections Among U.S. Residents Without International Travel. HAN Arch. 2021;(CDCHAN-00439):0-5. 
producing strains isolated from imported cases of enteric fever in England and Wales reveal multiple chromosomal integrations of bla CTX-M-15 in XDR Salmonella Typhi . J Antimicrob Chemother. 2021;1-8.

21. Octavia S, Chew KL, Lin RT, Teo JW. Azithromycin-Resistant $<$ em $>$ Salmonella enterica $</$ em $>$ Serovar Typhi AcrB-R717Q/L, Singapore. Emerg Infect Dis J [Internet]. 2021;27(2):624. Available from: https:/wwwnc.cdc.gov/eid/article/27/2/20-3874_article

22. Carey ME, Jain R, Yousuf M, Maes M, Dyson ZA, Thu TNH, et al. Spontaneous Emergence of Azithromycin Resistance in Independent Lineages of Salmonella Typhi in Northern India. Clin Infect Dis. 2021;72(5):e120-7.

23. Andrews JR, Vaidya K, Bern C, Tamrakar Di, Wen S, Madhup S, et al. High Rates of Enteric Fever Diagnosis and Lower Burden of Culture-Confirmed Disease in Peri-urban and Rural Nepal. J Infect Dis. 2018;

24. Andrews JR, Barkume C, Yu AT, Saha SK, Qamar FN, Garrett D, et al. Integrating Facility-Based Surveillance with Healthcare Utilization Surveys to Estimate Enteric Fever Incidence: Methods and Challenges. J Infect Dis. 2018;218(Suppl 4):S268-76.

25. Barkume C, Date K, Saha SK, Qamar FN, Sur Di, Andrews JR, et al. Phase i of the Surveillance for Enteric Fever in Asia Project (SEAP): An Overview and Lessons Learned. J Infect Dis. 2018;218(Suppl 4):S188-94.

26. Andrews S. Babraham Bioinformatics - FastQC A Quality Control tool for High Throughput Sequence Data. Soil. 1973.

27. Ewels P, Magnusson M, Lundin S, Käller M. MultiQC: Summarize analysis results for multiple tools and samples in a single report. Bioinformatics. 2016;

28. Wood DE, Lu J, Langmead B. Improved metagenomic analysis with Kraken 2. Genome Biol. 2019;

29. Yoshida CE, Kruczkiewicz P, Laing CR, Lingohr EJ, Gannon VPJ, Nash JHE, et al. The salmonella in silico typing resource (SISTR): An open web-accessible tool for rapidly typing and subtyping draft salmonella genome assemblies. PLoS One. 2016; genomic surveillance for public health and hospital microbiology labs. Genome Med.

554 31. Langmead B, Salzberg S. Bowtie2. Nat Methods. 2013; 
32. Li H, Handsaker B, Wysoker A, Fennell T, Ruan J, Homer N, et al. The Sequence Alignment/Map format and SAMtools. Bioinformatics. 2009;

33. Wong VK, Baker S, Connor TR, Pickard D, Page AJ, Dave J, et al. An extended genotyping framework for Salmonella enterica serovar Typhi, the cause of human typhoid. Nat Commun. 2016;

34. Croucher NJ, Page AJ, Connor TR, Delaney AJ, Keane JA, Bentley SD, et al. Rapid phylogenetic analysis of large samples of recombinant bacterial whole genome sequences using Gubbins. Nucleic Acids Res. 2015;

35. Ingle DJ, Nair S, Hartman H, Ashton PM, Dyson ZA, Day M, et al. Informal genomic surveillance of regional distribution of Salmonella Typhi genotypes and antimicrobial resistance via returning travellers. PLoS Negl Trop Dis. 2019;

36. Rahman SIA, Dyson ZA, Klemm EJ, Khanam F, Holt KE, Chowdhury EK, et al. Population structure and antimicrobial resistance patterns of salmonella typhi isolates in Urban Dhaka, Bangladesh from 2004 to 2016. PLoS Negl Trop Dis. 2020;

37. Tanmoy AM, Westeel E, De Bruyne K, Goris J, Rajoharison A, Sajib MSI, et al. antimicrobial resistance. MBio. 2018;

38. Britto CD, Dyson ZA, Mathias S, Bosco A, Dougan G, Jose S, et al. Persistent circulation of a fluoroquinolone-resistant Salmonella enterica Typhi clone in the Indian subcontinent. J Antimicrob Chemother. 2020;

39. Pragasam AK, Pickard D, Wong V, Dougan G, Kang G, Thompson A, et al. Phylogenetic Analysis Indicates a Longer Term Presence of the Globally Distributed H58 Haplotype of Salmonella Typhi in Southern India. Clin Infect Dis. 2020;

40. Britto CD, Dyson ZA, Duchene S, Carter MJ, Gurung M, Kelly DF, et al. Laboratory and molecular surveillance of paediatric typhoidal Salmonella in Nepal: Antimicrobial resistance and implications for vaccine policy. PLoS Negl Trop Dis. 2018;

41. Dyson ZA, Thanh DP, Bodhidatta L, Mason CJ, Srijan A, Rabaa MA, et al. Whole large phylogenies. Bioinformatics. 2014; 
43. Letunic I, Bork P. Interactive Tree Of Life (iTOL) v4: recent updates and new developments. Nucleic Acids Res. 2019;

44. Rambaut A, Lam TT, Carvalho LM, Pybus OG. Exploring the temporal structure of heterochronous sequences using TempEst (formerly Path-O-Gen). Virus Evol. 2016;

45. Didelot X, Croucher NJ, Bentley SD, Harris SR, Wilson DJ. Bayesian inference of ancestral dates on bacterial phylogenetic trees. Nucleic Acids Res [Internet]. 2018 Dec 14;46(22):e134-e134. Available from: https://pubmed.ncbi.nlm.nih.gov/30184106

593 46. Drummond AJ, Rambaut A. BEAST: Bayesian evolutionary analysis by sampling trees. BMC Evol Biol. 2007;

595 47. Volz EM, Frost SDW. Scalable relaxed clock phylogenetic dating. Virus Evol [Internet]. 

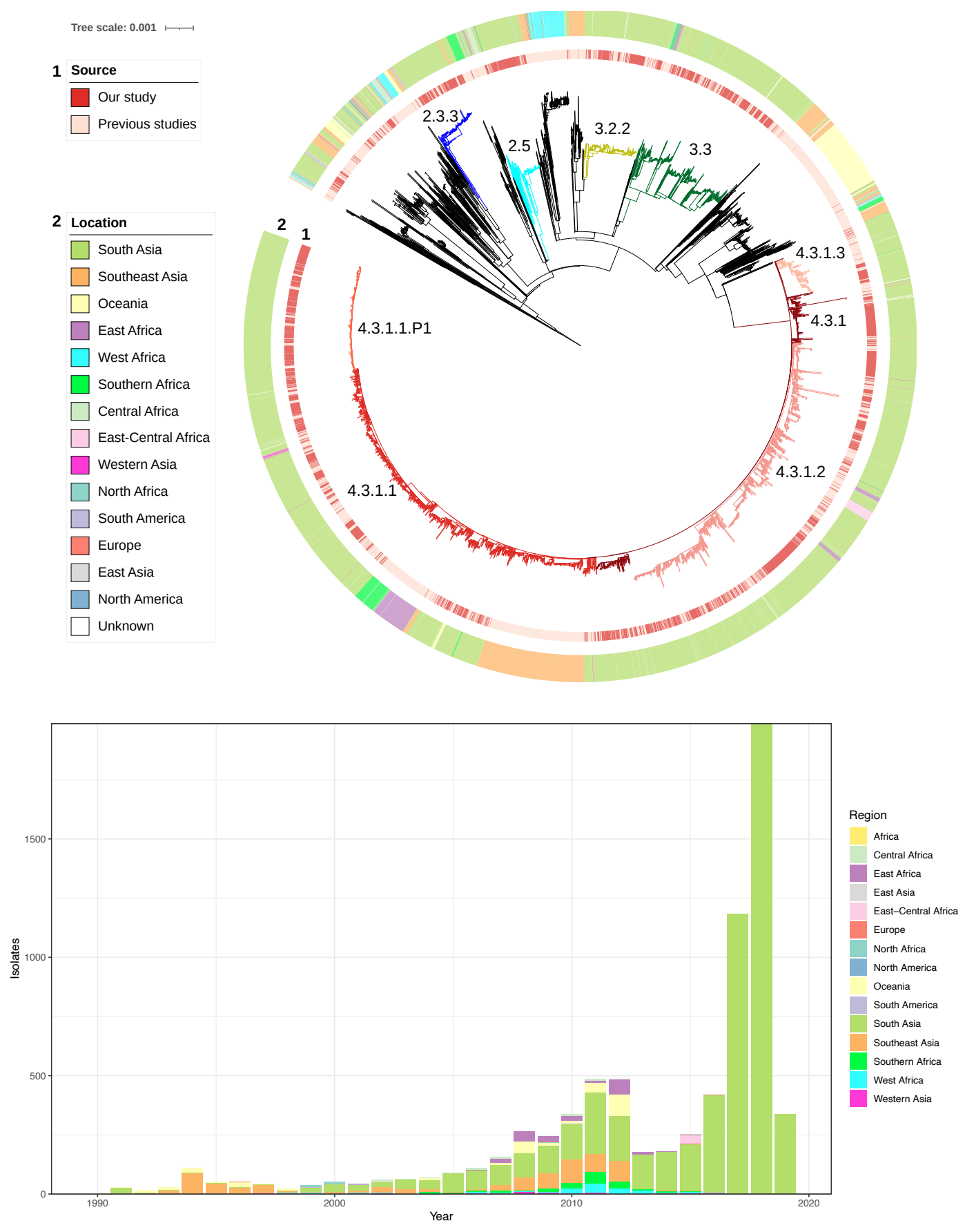

614 Figure 1. Global phylogeny of Salmonella Typhi. (a) Maximum likelihood tree of 7,658 S. Typhi 615 isolates from the global collection. Branch colors indicate the lineages 2.3.3 (blue), 2.5 (turquoise), 616 3.2.2 (yellow), 3.3 (green), 4.3.1 (dark red), 4.3.1.1 (red), 4.3.1.1.P1 (orange), 4.3.1.2 (pink), 617 4.3.1.3 (salmon) and other non-H58 (black). The inner ring indicates the source. The outer ring 618 indicates the region of isolation. The scale bar indicates nucleotide substitutions per site (b) 
medRxiv preprint doi: https://doi.org/10.1101/2021.09.03.21262852; this version posted September 7, 2021. The copyright holder for this preprint (which was not certified by peer review) is the author/funder, who has granted medRxiv a license to display the preprint in perpetuity.

It is made available under a CC-BY-NC-ND 4.0 International license .
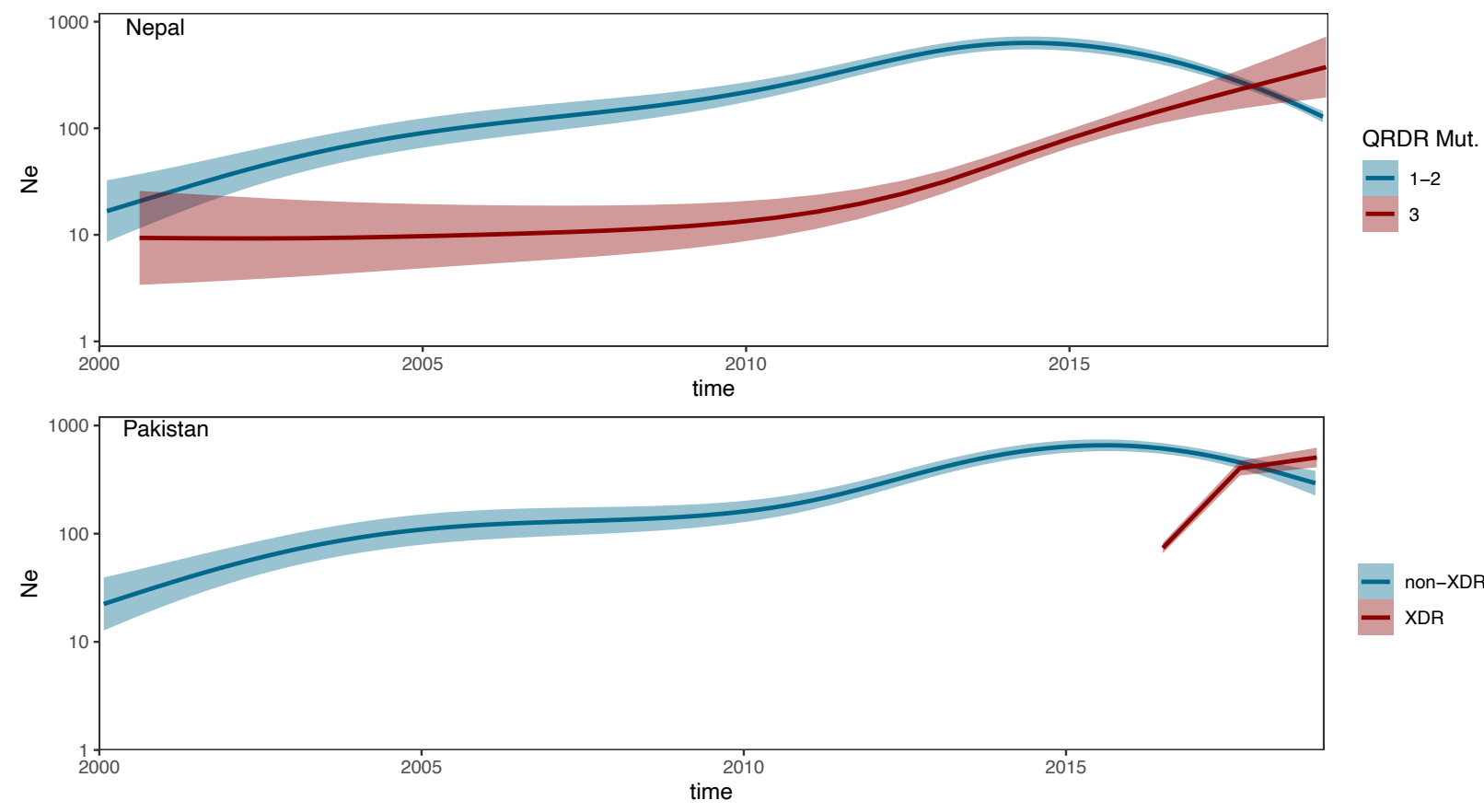

non-XDR

XDR

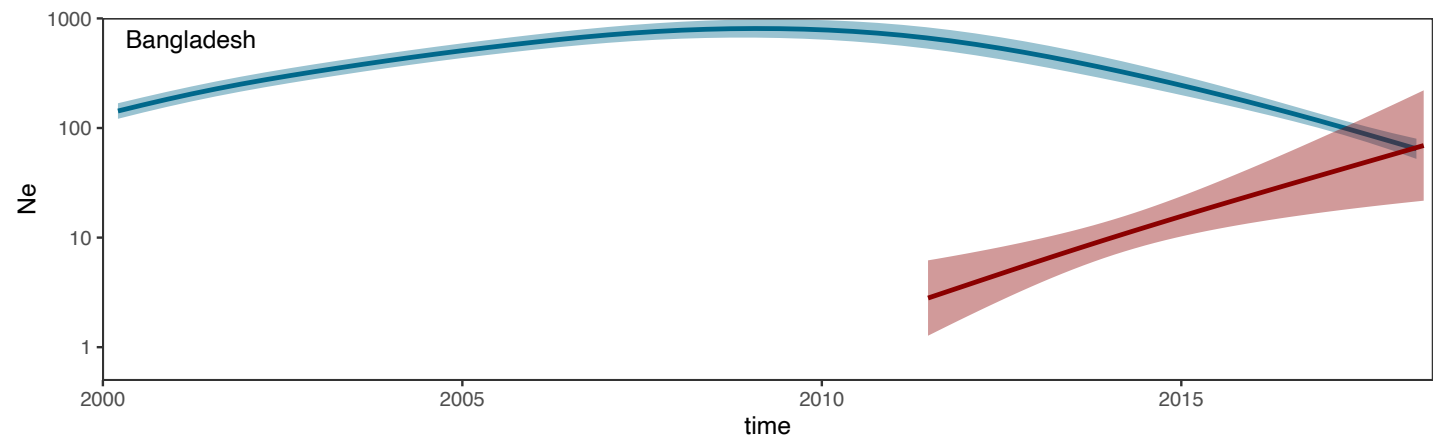

Azithro ${ }^{\mathrm{S}}$

Azithro $^{R}$

624 Figure 2. The effective population size $\left(\mathrm{N}_{\mathrm{e}}\right)$ of $\mathrm{H} 58$ lineages according to antimicrobial resistance 625 genotype in Nepal, Pakistan and Bangladesh. In Nepal, strains containing 1-2 mutations in the quinolone resistance determining region (QRDR) were compared with those containing 3

627 mutations. In Pakistan, extensively drug-resistant (XDR) strains were compared with non-XDR

628 strains. In Bangladesh, strains containing $\operatorname{acr} B$ mutations conferring azithromycin-resistance were

629 compared with those not containing the mutations. Light shading represents the $95 \%$ high

630 probability density (HPD) intervals of the estimates. 

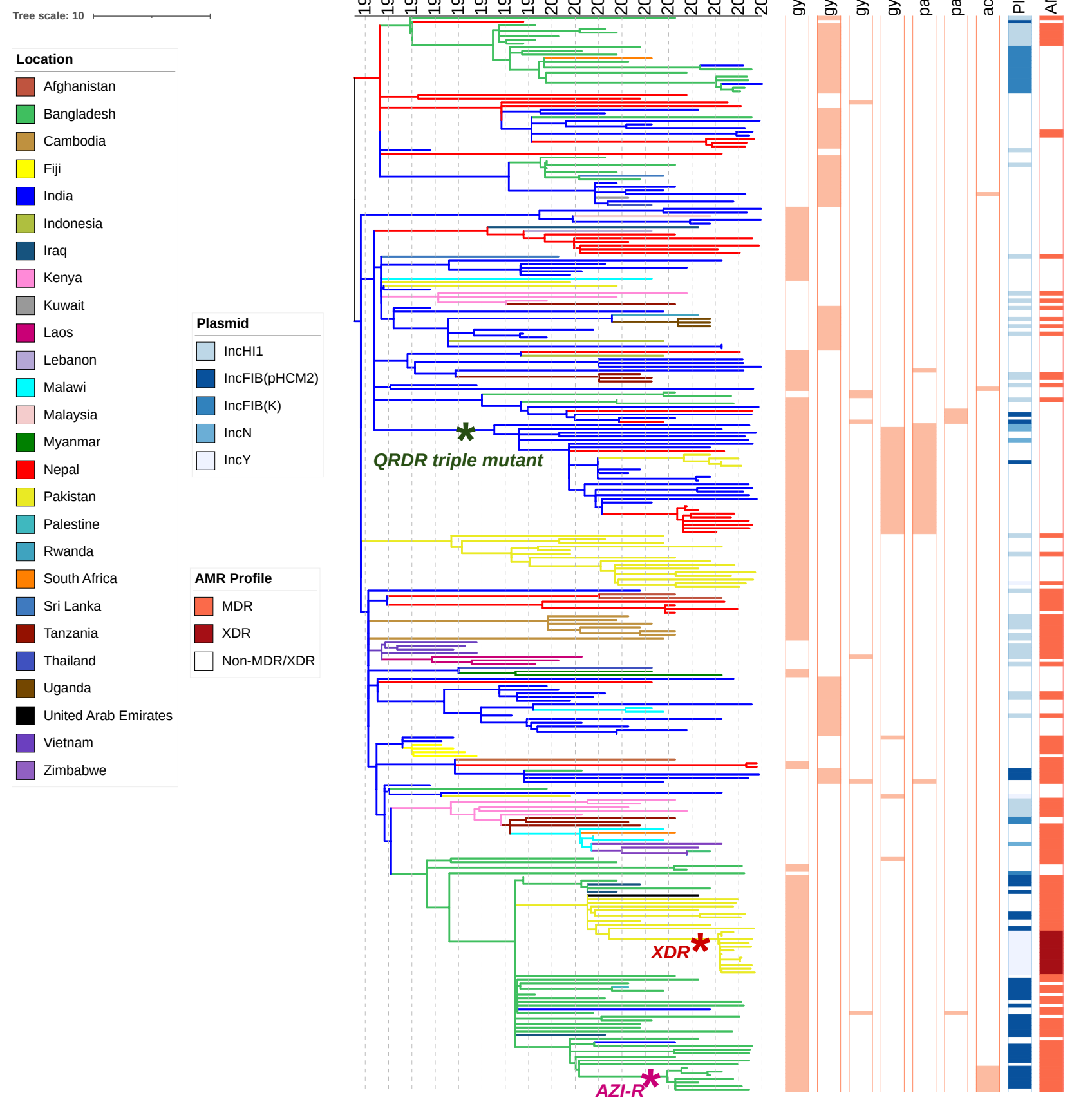

634 Figure 3. Phylogeography and global expansion of genotype 4.3.1 (H58) S. Typhi isolates. Timed

635 phylogenetic tree of genotype 4.3.1 S. Typhi isolates. The branch lengths are scaled in years and 636 are colored according to the location of the most probable ancestor of descendant nodes. The scale 637 bar indicates nucleotide substitutions per site. 


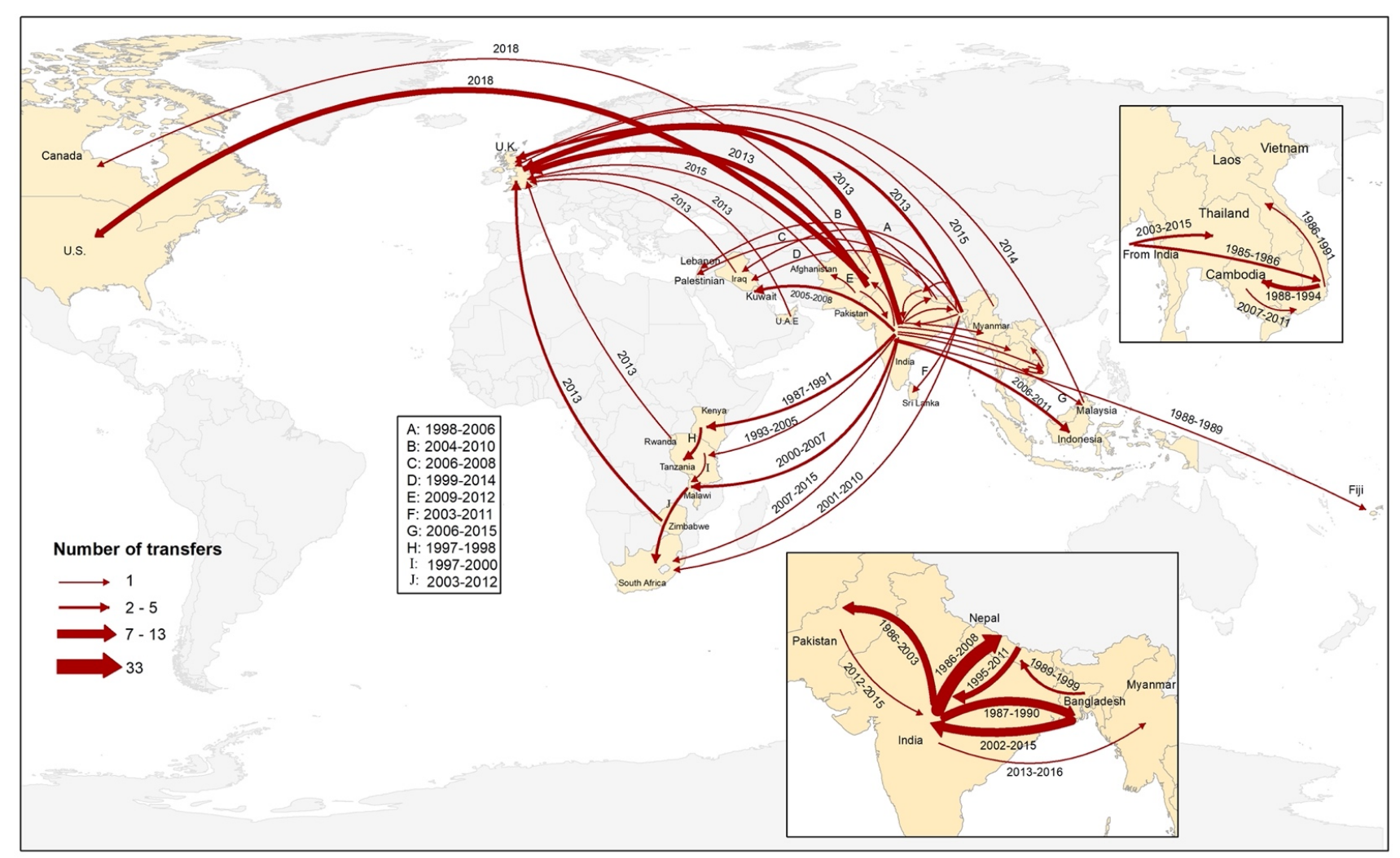

641 Figure 4. Geographical transfers within lineage 4.3.1 (H58) inferred from ancestral state

642 reconstruction of the timed phylogenetic tree. The size of each arrow is scaled to the estimated

643 number of transfers between the countries. Dates indicate the estimated first transfer between each

644 pair of countries. 
medRxiv preprint doi: https://doi.org/10.1101/2021.09.03.21262852; this version posted September 7, 2021. The copyright holder for this preprint (which was not certified by peer review) is the author/funder, who has granted medRxiv a license to display the preprint in perpetuity. It is made available under a CC-BY-NC-ND 4.0 International license .

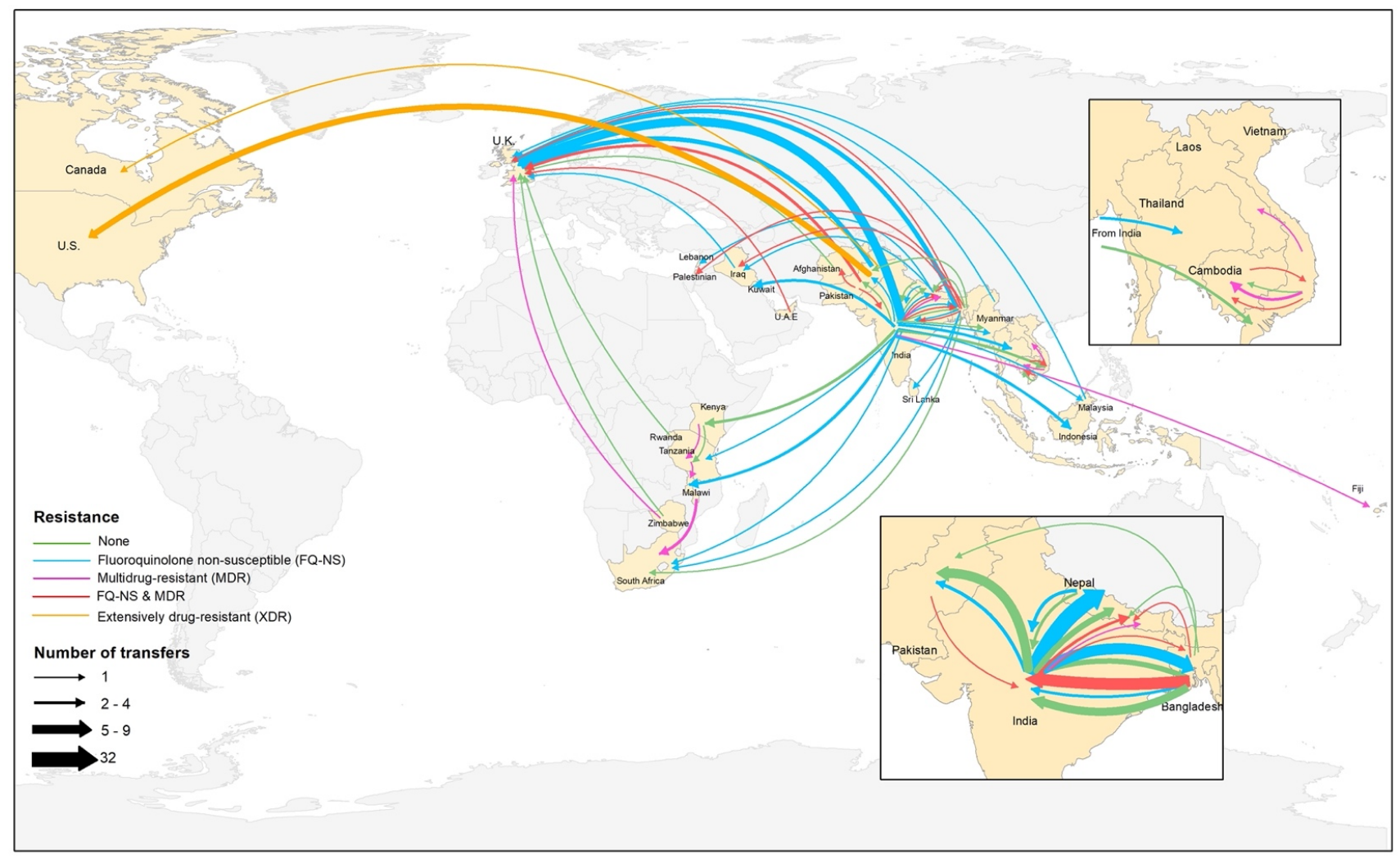

655 Figure 5. Major geographical transfers from 1990 onwards within the non-H58 and H58 lineages, 656 inferred from the phylogenetic trees. The size of each arrow indicates the relative number of likely 657 transfers between the countries. Arrow colors indicate antimicrobial resistance pattern. 